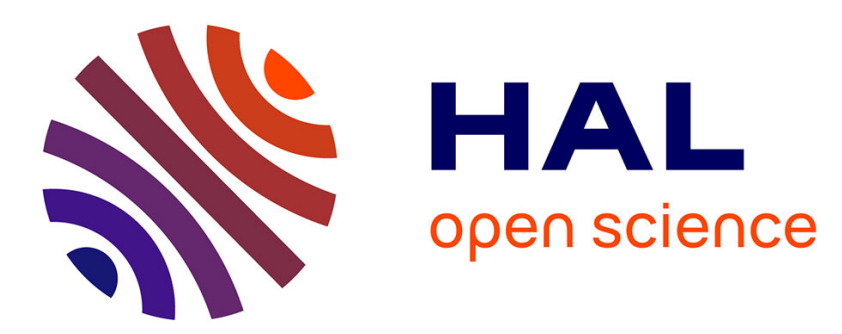

\title{
Ecological Dynamics: a theoretical framework for understanding sport performance, physical education and physical activity
}

\author{
Ludovic Seifert, Keith Davids
}

\section{- To cite this version:}

Ludovic Seifert, Keith Davids. Ecological Dynamics: a theoretical framework for understanding sport performance, physical education and physical activity. CS-DC'15 World e-conference, Sep 2015, Tempe, United States. hal-01291044

\author{
HAL Id: hal-01291044 \\ https://hal.science/hal-01291044
}

Submitted on 20 Mar 2016

HAL is a multi-disciplinary open access archive for the deposit and dissemination of scientific research documents, whether they are published or not. The documents may come from teaching and research institutions in France or abroad, or from public or private research centers.
L'archive ouverte pluridisciplinaire HAL, est destinée au dépôt et à la diffusion de documents scientifiques de niveau recherche, publiés ou non, émanant des établissements d'enseignement et de recherche français ou étrangers, des laboratoires publics ou privés. 


\title{
Ecological Dynamics: a theoretical framework for understanding sport performance, physical education and physical activity
}

\author{
Ludovic Seifert $^{1}$, Keith Davids ${ }^{2}$ \\ ${ }^{1}$ CETAPS - EA 3832, Faculty of Sport Sciences, University of Rouen, France \\ ${ }^{2}$ Centre for Sports Engineering Research, Sheffield Hallam University, Sheffield, UK
}

\begin{abstract}
This paper focuses on the role of Ecological Dynamics as a theoretical framework for analysing performance of athletes and sports teams as complex adaptive systems. It combines key concepts from ecological psychology and nonlinear dynamical system theory, seeking to enhance understanding of performance and learning contexts in sport, to aid the acquisition and transfer of adaptive human behaviours. In ecological psychology the continuous regulation of human behaviour is predicated on the role of information that emerges from the individual-environment system to guide activity. The information sources that constrain performance behaviours are affordances, which provide invitations for action offered by each individual's perception of functional relations with a performance environment. This information-based approach has been enhanced with the integration of tools and concepts from nonlinear dynamics to explain how information is cyclically related to the dynamics of a performance environment. Dynamical systems theory addresses the emergence of coordination tendencies that exist between and within components and levels of complex neurobiological systems. Ecological Dynamics identifies key properties of expertise in sport predicated on the performer-environment relationship as the appropriate scale of analysis. This paper introduces the key properties of expert movement systems that include multi- and meta-stability, the functional role of adaptive movement variability, redundancy, degeneracy and the attunement to affordances. Additionally, we discuss the concept of representative design, which in an ecological dynamics framework underpins the organisation of experimental and learning environments so that observations and acquired skills can be linked to emergent functional behaviours in a specific performance context.
\end{abstract}

Key words: coordination dynamics, adaptive variability of movement, affordances, representative design.

\section{Introduction}

The Ecological Dynamics framework sustains a scientific approach to studying the behaviours of neurobiological systems, especially processes of action, perception and cognition. Kugler and Turvey [1] considered that "Ecological science....is a multidisciplinary approach to the study of living systems, their environments and the reciprocity that has evolved between the two.....Ecological Psychology... [emphasizes] the study of information transactions between living systems and their environments, especially as they pertain to perceiving situations of significance to planning and executing of purposes activated in an environment".

In line with this philosophical direction, ecological dynamics has provided a systems orientation for the theoretical analysis of behaviours of athletes and sports teams over different timescales (performance, learning and development), conceptualised as complex adaptive systems [2]. In this research endeavour, we have sought to integrate key concepts from ecological psychology and nonlinear dynamics, in order to enhance understanding of 
performance, learning and expertise acquisition in sport. As we note later in this paper, the latter two processes are developed through the design of practice task simulations which exploit the transfer (from practice to competition) of adaptive human behaviours [3-5].

A major aspect of ecological dynamics is James Gibson's theory of ecological psychology, at the heart of which is 'systems thinking' [6], emphasising continuous organism-environment interactions as the relevant scale of analysis for understanding human behaviours in performance environments (e.g. an athlete-performance environment relationship). The central ideas of Gibsonian theorising, with respect to the directness of perception, have been linked back to the philosophical influence of Aristotle. In ecological psychology the continuous regulation of human behaviour is predicated on the role of information (e.g., optical, proprioceptive, haptic) that emerges from the individual-environment system to continuously guide activities like dribbling with a ball or intercepting a moving target. This conceptualisation of information differs greatly from traditional psychological connotations, which regarded that sensory systems yielded information from an environment, which was ambiguous and impoverished, needing interpretation with cognitive processes such as memory, attention and anticipation. In contrast, ecological psychology emphasises that knowledge of an environment underpins actions and is directly gained through perception without mediation by internalised representations [7]. Gibson's theory of direct perception proposes that perception is an act, emphasising that it is an active process in which perceptual and action systems function in a highly integrated and cyclical manner [6]. A key concept of ecological psychology, which we elucidate further, is affordances, which are opportunities or invitations for action that performers are surrounded by in a performance environment, becoming attuned to them through learning and experience $[6,8]$. These ideas from ecological psychology have been interpreted to suggest that with extensive practice and experience, an individual and a performance environment become ever more integrated as dynamical, selforganising systems coupled by information $[5,9]$. In these complementary systems intentional behaviours emerge from a process of exploration and learning before being stabilised into functional action patterns [9]. Therefore, in a performance environment, the most significant information sources that constrain athlete behaviours are affordances, which provide invitations or opportunities for action offered by each individual's perception of functional relations with a performance environment $[6,8]$.

This theory of ecological psychology has been enhanced with the integration of tools and concepts from nonlinear dynamics to explain how information is cyclically related to the dynamics of a performance environment [10]. Dynamical systems theory which provides a conceptual framework for understanding the emergence of coordination tendencies that exist between, and within, system components and levels of complex neurobiological systems (e.g. in human beings and sports teams) at multiple levels (e.g., from behaviour to brain)[10-12] with roots in thermodynamics [13] and synergetics [14]. Physical principles and concepts from nonlinear, dissipative, self-organizing systems explain coordination dynamics as a natural process of pattern formation in neurobiological systems. In particular, stability in neurobiological complex systems is ensured by a constant flow of energy, consisting of the exchanges of information between the system and its environment [14]. More broadly, the constant exchange of matter, energy, or information through continual and circular interactions between a system and its environment revealed self-organisation, i.e., assemblage and implementation of the components by the system itself without prescription from a higher-order system [14, 15]. Therefore, those systems are called dissipative dynamical systems [15]. From there, dynamical systems theory seeks to explain and predict how patterns of coordination emerge, adapt, persist, and change in humans considered as integrated complex systems [10]. Organisation in neurobiological systems is both facilitated and bounded by interacting constraints which shape the dynamics of emergent behaviours [16]. 
Constraints on behaviours include task, performer and environmental factors. The unpredictable nature of the environment and the many individual differences that exist in personal characteristics and functions of athletes signify that indeterminate solutions exist for many tasks in sport. This idea emphasizes the circular causality of the relationship between each individual and a performance environment, mediated by brain and behaviour, and processes of perception and action [10]. The causality between brain and behaviour, and between processes of perception and action, is not linear but cyclical [6], as the individual continuously constructs goal-directed interactions with a performance environment. From this perspective, expertise is the continuous functional adaptation of behaviours to a set of interacting constraints in order to exploit them to the fullest in achieving specific intended performance goals [17].

Therefore by articulating Ecological psychology theory and Dynamical Systems theory, Ecological Dynamics presents a viable theoretical framework for studying sport performance because it's systems oriented perspective on the relationship between each individual and a performance environment addresses the weaknesses of traditional approaches to expert performance in sport, which separately focus on the performer and the environment. From there this paper presents the ecological dynamics theoretical rationale to identify key properties of expertise in sport, predicated on the performer-environment relationship as the appropriate scale of analysis. Indeed, Seifert et al. [17] previously observed that, with this theoretical orientation, key properties of expert movement systems that are worthy of further investigation include multi- and meta-stability [18], adaptive variability according to interacting constraints [19, 20, 16], redundancy, degeneracy [21-24], and the attunement to affordances $[6,25]$. From there, our presentation is organised in four sections and discusses: (1) the functional role of movement variability, which could be exhibited by multi- and metastability of movement patterns, reflecting neurobiological degeneracy properties; (2) the interest of representative learning and training design to guarantee ecological validity, generalizability and skill transfer; (3) the affordances picking-up and the attunement and calibration to specifying information for action; (4) all together, these properties are the basis for a non-linear pedagogy. Indeed, this last section about non-linear pedagogy [27] argues the idea that skill acquisition does not emerge from the internal representation of declarative and procedural knowledge, or the imitation of expert behaviours to linearly reduce a perceived 'gap' separating movements of beginners and a putative expert model. Rather skill acquisition and expert performance correspond with the on-going co-adaptation of an individual's behaviours to dynamically changing, interacting constraints, individually perceived and encountered $[17,26]$.

\section{The functional role of movement variability}

Developing knowledge of the functional role of movement variability is essential to understanding expert performance in many different sports (involving individuals and teams; ball games and outdoor activities; land and aquatic environments) $[19,28,29]$. Studying the functional role of movement variability involves assessing how adaptive human behaviour is by analysing the balance between movement pattern stability (i.e., persistent behaviour) and flexibility (i.e., variable behaviour) relative to a performance context $[9,17]$. Specifically, our work has sought to explore how individuals adapt their motor behaviours in various performance contexts, i.e., when the environmental properties are stable or in unstable or transitional regions where movement patterns co-exist (technically known as a metastable region; [30]). According to Kelso [30], meta-stability is the "simultaneous realisation of two competing tendencies: the tendency of the components to couple together and the tendency for the components to express their intrinsic independent behaviour" (p.186). In a meta-stable 
performance region, component tendencies of independence co-exist, explaining how rich and varied movement patterns can spontaneously emerge in dynamic sport environments as an individual adapts his/her motor behaviours to achieve particular performance goals. These continuous changes of motor behaviours could merely result in an adaptive pattern refinement or the adoption of new form through a non-linear phase transition. There exists some evidence of meta-stability in skilled sport performance, such in boxing [31] and cricket [32]. For example, Hristovski et al. [31] investigated how boxers' striking patterns were adapted when they punched a heavy-bag at various scaled distances to the target. At greater distances from the boxing bag, 'jab' movement pattern emerged, whereas at closer distances, 'uppercuts' or 'hooks' patterns were observed. At a critical intermediated distance, the novice boxers explored a rich, varied and creative range of movement patterns involving 'uppercuts', 'hooks' and 'jabs', which characterised meta-stable performance region [31].

In sum, key properties of complex, dynamical systems concern the continuous formation of stable behavioural patterns (i.e., attractors); non-linear transitions from one attractor to another attractor (e.g., system transitions or bifurcations, hysteresis, critical slowing down); multi-stability (i.e., ability to transit between multiple states of organisation under given constraints); meta-stability (i.e., ability to exploit co-existing coordination tendencies in a transition or unstable region); and variability (exploiting critical fluctuations to enable adaptive behavioural transitions) [10, 17]. In our research programme, we have investigated how athletes perform different types of movement and/or to adopt one of a number of coexisting modes of coordination between system components, in order to achieve the same functional performance outcomes, predicated on the neurobiological system property of degeneracy. Edelman and Gally [21] defined degeneracy as the capacity of system components that differ in structure to achieve the same function or performance output. This structural property in humans indicates the availability of an abundance of motor system degrees of freedom, which can take on different roles when assembling functional actions during sport performance. These key properties signify that, in sport performance, although basic movement patterns need to be acquired by developing athletes, there exists no ideal movement template towards which all learners should aspire, since relatively unique functional movement solutions emerge from the interaction of key constraints [16].

Newell [16] defined three types of constraints: environmental, task and organismic (personal). Personal constraints are structural or functional and refer to characteristics of an individual such as genes, anthropometric properties, cognition, motivation, and emotions. In swimming, personal constraints may include passive drag and flotation parameters (hydrostatic lift, sinking force acting at the ankle) that could be artificially modified by wearing a wet suit and influence movement and coordination organisation [33]. Other body characteristics like strength, endurance and laterality (handedness and the preferred breathing side) can also influence movement and coordination organisation [33]. Environmental constraints are external to an individual and can be physical, reflecting the environmental conditions of the task. For instance, in climbing, temperature and altitude can make the ascent harder. In particular, it is well known that a high ambient temperature can cause swelling in the feet, which means that climbing becomes uncomfortable. Humidity can cause a climber's hands to become sweaty, which challenges the graspability of holds. In swimming, environmental constraints refer to water properties such as the temperature and density and viscosity of the fluid, the direction and type of water flow (laminar vs. turbulent, quantified with Reynolds number), underwater visibility, and waves on the surface of the water (e.g. assessed with Froude number) [33]. Task constraints include the goal of the task, the rules, boundary locations, instructions or equipment specifying a response. For instance, among these task constraints, Vilar et al. [34] showed that the cooperative (within team) or competitive 
(between teams) nature of the interpersonal coordination in team sport influences the way of how individuals interact to reach the task-goal.

\section{Representative learning and training design: a condition for skill transfer}

In an ecological dynamics framework, the concept of representative design underpins the organisation of experimental and learning environments so that observations and acquired skills can be linked to emergent functional behaviours in a specific performance context [35]. The representative design framework provides guidance for the development of ecological constraints that best reflect continuous performance interactions of athletes and performance environments, and the role of variability during these interactions. This is an important feature of skill transfer in complex adaptive systems, since it ensures that cognitions, perceptions and actions used to regulate behaviour in one performance context (e.g., a practice environment) can be expected to generalise and underpin performance in another context (competitive performance environments) [36]. Skill transfer can be characterised as positive (i.e. performance is improved under different constraints than would otherwise be the case without learning), neutral, or negative (i.e. performance decreases under different constraints than would otherwise have been the case without learning) [37, 38]. Positive transfer can occur when the existing intrinsic dynamics (i.e. performance disposition or tendencies) of an individual cooperate with the dynamics of a new task to be learned, facilitating successful performance behaviours [39]. Negative transfer occurs when intrinsic and task dynamics compete [39]. Cooperation and competition phenomena are influenced by the intrinsic dynamics of the individual which are modifiable in terms of structural and functional adaptations, many of which that are specific to, and can be shaped by, constraints on experience. An important research challenge is to effectively characterise different performance ecologies along each axis of transfer (near and far), in order to predict how processes of skill transfer might support performance (positive, neutral or negative adaptation of the intrinsic dynamics). Near domain transfer refers to when skills are generalised to a new set of performance constraints that, although different, maintain interactions among key system components [37, 38]. Far transfer refers to an across-domain transfer (i.e., domain scaled) where separate subsystems become important in different performance environments (e.g., when involving qualitatively different decision making processes, physiological or psychological performance aspects). In sum, the relationship between two performance environments is captured by numerous, dynamic and interacting constraints: environmental, task and personal (both structural e.g., strength, flexibility, height, and functional e.g., decision making, attunement and calibration to specifying information for action) [3, 4]. This makes it difficult to predict transferability of movement behaviours between two performance domains. In our programme of research we have sought to undertake necessary theoretical and empirical developments to enhance understanding of this important process.

According to our analysis of key ideas in ecological dynamics, transferability that enhances functional skill adaptations might relate to specificity and generality of transfer processes induced by the selection and/or design of a performance environment. This is because the design of some practice task constraints simulate the properties of performance environment more specifically than others [40]. An ecological dynamics framework suggests that the level of specificity between performance environments relates to the ecological niche of an individual [6], providing a landscape of available affordances that supports emergence of functional adaptive behaviours [41]. The issue of generality or specificity of transfer particularly relates to the capability of an individual to pick up and use these affordances under different performance conditions. Bruineberg and Rietveld [41] defined the landscape of affordances as the affordances available in an ecological niche, i.e., the whole spectrum of 
abilities available in our socio-cultural practices. The richness of the landscape of affordances raises questions over the nature of learning designs that can help an individual to pick up only relevant affordances in a given performance environment, i.e., through attunement and calibration to specifying information for adaptive behaviours [25].

\section{Attunement to affordances}

An important concern is the information that guides behaviours of complex systems, emphasized through the role of affordances as a key property of performer-environment interactions [6]. Affordances are particular properties of a performance environment, which are perceived in 'animal-relevant' terms, i.e., what they offer, invite or demand of an organism in terms of actions [8]. The concept of affordances provides a powerful way of understanding how processes of perception and action function in complex adaptive systems, since "within the theory of affordances, perception is an invitation to act, and action is an essential component of perception" (p46) [6]. Affordances are defined by the complementary relations between an individual and an environment. They are both objective and subjective to each performer, since they are ecological properties of the environment picked up relative to an individual $[42,43]$. Affordances for action in an environment are specified within a unique frame of reference for each individual performer, whether learner or expert, adult or child. Descriptions of the state of the environment are 'frame dependent' because affordances for action are perceived relative to relevant properties of an individual including the scale of key body dimensions (e.g., height, limb sizes). For example, when dribbling in team games, affordances might include angles and distances to and distances between approaching defenders [44, 45]. Ecological dynamics rationalises that such distance or angular values are not perceived in arbitrary metric scales, such as different units of measurement (e.g. yards or metres). Rather, gaps, distances, angles, heading orientation afford different actions from different performers based on their personal constraints [16]. Such personal constraints can be structural (e.g., height and weight and limb length of dribblers) or functional (speed, movement time, power, technical skill in dribbling a ball). In order to negotiate the environment with tools, as extensions of their bodies, during functional behaviours, research has verified theoretical ideas that individual performers perceive different affordances with respect to their own morphological and biomechanical properties, as well those properties in other performers (e.g., defenders). Athletes need to be able to learn how to solve particular performance problems by picking up relevant body-scaled environmental properties, such as whether their speed in dribbling with the ball can affords passing through gaps of varying dimensions on field, with respect to their relevant physical organismic constraints.

From a Gibsonian philosophical orientation, learning in neurobiology involves the pick up of affordances. Gibson [6] proposed that all organisms perceived affordances, which can constrain their actions. Skilled individuals become progressively attuned available affordances as their expertise levels increase [25]. Affordances are action opportunities invited from an individual, which are predicated on knowledge of a performance environment (rather than knowledge about) [6,7]. This type of knowledge highlights the importance of adopting a person-environment scale of analysis in an ecological dynamics orientation. Basically, it is argued that the environment is composed of physical properties (such as light amplitudes or surface hardness) and the individual is made up of measurable action capabilities. The relationship between the physical properties of the environment and the individuals action capabilities constitutes an affordance [8]. In climbing, affordances refer to "climbing opportunities" [46], i.e., environmental properties that invite hold reach-ability, grasp-ability and climb-ability. In particular, in rock climbing, Boschker, Bakker and Michaels [46] demonstrated how experts recalled more information specifying the functional 
properties of a climbing wall (knowledge of a performance environment), neglecting to perceive its structural features (knowledge about a performance environment). Conversely, novices were not able to recall such functional properties of the wall to support their actions and they tended to report almost exclusively the structural (less functional) features of the holds [46]. For instance, if a rock climber grasps a surface hold because of its large size, instead of its shape or its orientation, he/she may be using the wrong structural feature (e.g., hold size instead of hold shape or hold orientation) to decide which hold to grasp and how to grasp it [47]. An affordance in rock climbing, specifies what a hold is and what a hold means, not separately, but unified in one perceiving-acting process. Perceiving opportunities for specific actions requires perceptual attunement to and calibration of relevant informational variables, meaning that individuals need to pick up a range of perceptual variables from different system modalities (haptic, kinesthesis, auditory, visual) that specify a relevant property of a performance environment $[25,48]$. The term 'relevant' signifies functionality, as this property enables an individual performer to achieve a specific task goal with efficacy. An important characteristic of experts is their perceptual attunement to relevant informational variables, revealing that experts are better at perceiving affordances than beginners.

\section{Nonlinear pedagogy}

The previous key theoretical concepts in ecological dynamics provide a powerful underlying principled philosophical basis for the implementation of a nonlinear pedagogy [27]: a pedagogical framework for enhancing learning in athletes and sports teams as complex adaptive systems. Chow et al. [27] proposed several principles for a nonlinear pedagogy in sport and physical education: the representativeness of the situation, focus of attention, functional variability through exploratory behaviours, manipulation of constraints, and ensuring relevant information-movement couplings (e.g., favouring perception of affordances). Through these principles, nonlinear pedagogy emphasizes the non linearity and non proportionality between the amount of practice undertaken and the skill acquisition (i.e., the level of expertise) [27]. This philosophical orientation to practice contrasts completely with ideas from the theory of deliberate practice, which are beginning to be discredited in the field of expertise acquisition $[49,50]$. In contrast to such a linear pedagogical orientation that prescribes the movement to be learned, giving numerous verbal instructions, nonlinear pedagogy respects and even enables the use of the degenerate nature of neurobiological systems, encouraging behavioural exploration, interaction with task and environmental constraints [51, 52]. This theoretical approach to learning design requires a respect for processes like task simplification rather than part-task decomposition, in the sense that learning and training designs have to preserve the complexity of an activity to maintain the coupling between information and movement [4]. In nonlinear pedagogy, error-reduction towards a specific model is thus not relevant. The challenge for coaches, instructors and physical education teachers is instead to create conditions that facilitate the exploratory process for the performer, rather than merely providing a precise description and prescription of a movement pattern or a team tactical pattern to follow. For instance, key aspect of nonlinear pedagogy is to use external focus of attention instructions to enhance a more sub conscious control of movement with attention paid to the effects of a movement on the environment, rather than the precise form of the movement, which seems to encourage the acquisition of important affordable in skill learning.

\section{Conclusion}

In summary, an ecological dynamics framework provides a unique philosophical orientation, which can address current challenges in understanding sport performance and skill acquisition 
in athletes and sports teams considered as complex adaptive systems. Our research programme has shown that addressing these challenges can enhance the effectiveness of current understanding and practice in sport science, pedagogical practice and performance analysis. In our work, our specific aims have included:

- $\quad$ To identify key properties of expertise and skill acquisition in athletes,

- $\quad$ To enhance understanding of how to design affordances into practice environments in sport which simulate competitive performance environments,

- $\quad$ To understand the functional and adaptive role of movement variability (within and between individuals),

- To help to design representative and interactive task constraints in sports development programmes, facilitating an individualised approach,

- $\quad$ Engineering technology and equipment to enhance skill acquisition during practice and training, predicated on complexity and neurobiological principles,

- $\quad$ To apply principles of a non-linear pedagogy to re-shape current Physical Education and Coaching practices.

\section{Scientific Validation}

This paper has been unanimously validated in a collaborative review mode with the following reviewers:

- Ana Sousa, University of Porto, Faculty of Sport, Porto, Portugal.

- María Carmen Lemos, University of Sevilla, Department of Condensed Matter Physics, Sevilla, Spain.

- Carlota Torrents Martin, National Institute of Physical Education (INEFC), Barcelona, Spain.

\section{Acknowledgments}

This project received the funding of the CPER/GRR Logistic Mobility Numeric XTerm.

\section{Bibliography}

1. Kugler, P.N., Kelso, J.A.S., Turvey, M.T.: On the Concept of Coordinative Structures as Dissipative Structures: I. Theoretical Lines of Convergence. Adv. Psychol. 1, 3-47 (1980).

2. Davids, K., Hristovski, R., Araújo, D., Balague Serre, N., Button, C., Passos, P.: Complex systems in sport. Routledge, Taylor \& Francis Group, London, UK (2014).

3. Davids, K., Araújo, D., Hristovski, R., Passos, P., Chow, J.Y.: Ecological dynamics and motor learning design in sport. In: Hodges, N.J. and Williams, A.M. (eds.) Skill acquisition in Sport: research, theory and practice. pp. 112-130. Routledge (taylor and francis Group), New York, NY, USA (2012).

4. Davids, K., Button, C., Bennett, S.J.: Dynamics of skill acquisition: A Constraints-led approach. Human Kinetics, Champaign, IL (2008).

5. Araújo, D., Davids, K., Hristovski, R.: The ecological dynamics of decision making in sport. Psychol. Sport Exerc. 7, 653-676 (2006). 
6. Gibson, J.: The ecological approach to visual perception. Houghton Mifflin, Boston (1979).

7. Davids, K., Araújo, D.: The concept of 'Organismic Asymmetry' in sport science. J. Sports Sci. 13, 633-40 (2010).

8. Withagen, R., de Poel, H.J., Araújo, D., Pepping, G.-J.: Affordances can invite behavior: Reconsidering the relationship between affordances and agency. New Ideas Psychol. 30, 250-258 (2012).

9. Warren, W.H.: The dynamics of perception and action. Psychol. Rev. 113, 358-89 (2006).

10. Kelso, J.A.S.: Dynamic Patterns: the self-organization of brain and behavior. MIT, Cambridge, MA (1995).

11. Jantzen, K.J., Oullier, O., Kelso, J.A.S.: Neuroimaging coordination dynamics in the sport sciences. Methods. 45, 325-35 (2008).

12. Zanone, P.G., Kostrubiec, V.: Searching for (Dynamic) principles of learning. In: Jirsa, V.K. and Kelso, J.A.S. (eds.) Coordination Danymics: issues and trends. pp. 58-89. Springer, Berlin (2004).

13. Prigogine, I., Stengers, I.: La nouvelle alliance [The new alliance]. Gallimard, Paris, FR (1979).

14. Haken, H.: Synergetics: an introduction: nonequilibrium phase transition and selforganization in physics, chemistry, and biology. Springer (1983).

15. Prigogine, I.: Les lois du chaos. Flammarion, Paris (1994).

16. Newell, K.: Constraints on the development of coordination. In: Wade, M. and Whiting, H. (eds.) Motor development in children: Aspects of coordination. pp. 341361. Martinus Nijhoff Publishers, Amsterdam (1986).

17. Seifert, L., Button, C., Davids, K.: Key properties of expert movement systems in sport : an ecological dynamics perspective. Sports Med. 43, 167-78 (2013).

18. Kelso, J.A.S.: Multistability and metastability: understanding dynamic coordination in the brain. Philos. Trans. R. Soc. Lond. B. Biol. Sci. 367, 906-18 (2012).

19. Davids, K., Bennett, S.J., Newell, K.M.: Movement System Variability. Human Kinetics, Champaign, Illinois. (2006).

20. Newell, K.M., Corcos, D.M.: Variability and motor control. Human kinetics, Champain, IL (1993).

21. Edelman, G.M., Gally, J. a: Degeneracy and complexity in biological systems. Proc. Natl. Acad. Sci. U. S. A. 98, 13763-8 (2001). 
22. Mason, P.H.: Degeneracy at Multiple Levels of Complexity. Biol. Theory. 5, 277-288 (2010).

23. Whitacre, J.M.: Degeneracy: a link between evolvability, robustness and complexity in biological systems. Theor. Biol. Med. Model. 7, 6 (2010).

24. Price, C.J., Friston, K.J.: Degeneracy and cognitive anatomy. Trends Cogn. Sci. 6, 416-421 (2002).

25. Fajen, B.R., Riley, M.R., Turvey, M.T.: Information, affordances, and the control of action in sport. Int. J. Sport. Psychol. 40, 79-107 (2009).

26. Seifert, L., Davids, K.: Intentions , Perceptions and Actions Constrain Functional Intraand Inter- Individual Variability in the Acquisition of Expertise in Individual Sports. Open Sports Sci. J. 5, 68-75 (2012).

27. Chow, J.Y., Davids, K., Hristovski, R., Araújo, D., Passos, P.: Nonlinear pedagogy: Learning design for self-organizing neurobiological systems. New Ideas Psychol. 29, 189-200 (2011).

28. Davids, K., Glazier, P.S., Araújo, D., Bartlett, R.M.: Movement systems as dynamical systems: the functional role of variability and its implications for sports medicine. Sports Med. 33, 245-60 (2003).

29. Glazier, P.S., Davids, K.: On analysing and interpreting variability in motor output. J. Sci. Med. Sport. 12, (2009).

30. Kelso, J.A.S.: Multistability and metastability: understanding dynamic coordination in the brain. Philos. Trans. R. Soc. Lond. B. Biol. Sci. 367, 906-18 (2012).

31. Hristovski, R., Davids, K., Araújo, D., Button, C.: Hox boxers decide to punch a target: emergent behaviour in nonlinear dynamical movement systems. J. Sci. Med. Sport. 5, 60-73 (2006).

32. Pinder, R.A., Davids, K., Renshaw, I.: Metastability and emergent performance of dynamic interceptive actions. J. Sci. Med. Sport. 1-7 (2012).

33. Seifert, L., Komar, J., Barbosa, T., Toussaint, H., Millet, G., Davids, K.: Coordination pattern variability provides functional adaptations to constraints in swimming performance. Sports Med. 44, 1333-45 (2014).

34. Vilar, L., Araújo, D., Davids, K., Button, C.: The role of ecological dynamics in analysing performance in team sports. Sports Med. 42, 1-10 (2012).

35. Brunswik, E.: Perception and the representative design of psychological experiments. University of California Press, Berkeley, CA, USA (1956).

36. Pinder, R.A., Davids, K., Renshaw, I., Araújo, D.: Representative learning design and functionality of research and practice in sport. J. Sport Exerc. Psychol. 33, 146-55 (2011). 
37. Issurin, V.: Training transfer: Scientific background and insights for practical application. Sport. Med. 43, 675-694 (2013).

38. Rosalie, S., Müller, S.: A model for the transfer of perceptual-motor skill learning in human behaviors. Res. Q. Exerc. Sport. 83, 413-421 (2012).

39. Zanone, P.G., Kelso, J.A.S.: Coordination dynamics of learning and transfer: collective and component levels. J. Exp. Psychol. Hum. Percept. Perform. 23, 1454-80 (1997).

40. Seifert, L., Wattebled, L., L'hermette, M., Bideault, G., Herault, R., Davids, K.: Skill transfer, affordances and dexterity in different climbing environments. Hum. Mov. Sci. 32, 1339-52 (2013).

41. Bruineberg, J., Rietveld, E.: Self-organization, free energy minimization, and optimal grip on a field of affordances. Front. Hum. Neurosci. 8, 599 (2014).

42. Scarantino, A.: Affordances explained. Philos. Sci. 70, 949-961 (2003).

43. Turvey, M., Shaw, R.: Ecological foundations of cognition I: Symmetry and specificity of animal-environment systems. J. Conscious. Stud. 65, 95-110 (1999).

44. Passos, P., Araújo, D., Davids, K., Gouveia, L., Serpa, S., Milho, J., Fonseca, S.: Interpersonal pattern dynamics and adaptive behavior in multiagent neurobiological systems: conceptual model and data. J. Mot. Behav. 41, 445-59 (2009).

45. Passos, P., Milho, J., Fonseca, S., Borges, J., Araújo, D., Davids, K.: Interpersonal distance regulates functional grouping tendencies of agents in team sports. J. Mot. Behav. 43, 155-63 (2011).

46. Boschker, M.S.J., Bakker, F.C., Michaels, C.F.: Memory for the functional characteristics of climbing walls: perceiving affordances. J. Mot. Behav. 34, 25-36 (2002).

47. Seifert, L., Orth, D., Hérault, R., Davids, K.: Affordances and grasping patterns variability during rock climbing. In: Davis, T., Passos, P., Dicks, M., and WeastKnapp, J. (eds.) Studies in Perception and Action XII: Seventeenth International Conference on Perception and Action. pp. 114-118. Psychology Press, Taylor \& Francis, Estoril, Portugal (2013).

48. Jacobs, D.M., Michaels, C.F.: Direct Learning. Ecol. Psychol. 19, 321-349 (2007).

49. Tucker, R., Collins, M.: What makes champions? A review of the relative contribution of genes and training to sporting success. Br. J. Sports Med. 46, 555-61 (2012).

50. Hambrick, D., Oswald, F., Altmann, E., Meinz, E., Gobet, F., Campitelli, G.: Deliberate practice: Is that all it takes to become an expert? Intelligence. 45, 34-45 (2014). 
51. Chow, J.Y., Davids, K., Button, C., Shuttleworth, R., Renshaw, I., Araújo, D.: The Role of Nonlinear Pedagogy in Physical Education. Rev. Educ. Res. 77, 251-278 (2007).

52. Chow, J.Y., Davids, K., Button, C., Shuttleworth, R., Renshaw, I., Araújo, D.:

Nonlinear pedagogy: a constraints-led framework for understanding emergence of game play and movement skills. Nonlinear Dynamics. Psychol. Life Sci. 10, 71-103 (2006). 